Tjalling C. Koopmans Research Institute Tplligh Aoopman

Discussion Paper Series nr:14-07

\title{
Soccer jersey sponsors and the world cup
}

Loek Groot

Joras Ferwerda 


\title{
Tjalling C. Koopmans Research Institute Utrecht School of Economics \\ Utrecht University
}

Kriekenpitplein 21-22

3584 EC Utrecht

The Netherlands

telephone $\quad+31302539800$

fax +31302537373

website www.koopmansinstitute.uu.nl

The Tjalling C. Koopmans Institute is the research institute and research school of Utrecht School of Economics.

It was founded in 2003, and named after Professor Tjalling C. Koopmans, Dutch-born Nobel Prize laureate in economics of 1975.

In the discussion papers series the Koopmans Institute publishes results of ongoing research for early dissemination of research results, and to enhance discussion with colleagues.

Please send any comments and suggestions on the Koopmans institute, or this series to L.M.vanDort@uu.nl

ontwerp voorblad: WR IK U trecht

\author{
How to reach the authors \\ Please direct all correspondence to the first author. \\ Loek Groot \\ J oras Ferwerda \\ Utrecht University \\ Utrecht School of Economics \\ Kriekenpitplein 21-22 \\ 3584 TC Utrecht \\ The Netherlands. \\ E-mail: L.F.M.Groot@uu.nl
}

This paper can be downloaded at: http: //

www.uu.nl/rebo/economie/discussionpapers 
Utrecht School of Economics

Tjalling C. Koopmans Research Institute

Discussion Paper Series 14-07

\title{
Soccer jersey sponsors and the world cup
}

\author{
Loek Groot \\ J oras Ferwerda
}

Utrecht School of Economics Utrecht University

April 2014

\begin{abstract}
The market for soccer jerseys is a multibillion market dominated by Adidas, Nike and Puma. This paper investigates whether jersey sponsorship has a non-arbitrary effect on the outcomes of World Cup knockout matches. The results show that in the knockout stages of the last four World Cup tournaments, especially national teams sponsored by Adidas perform significantly better than expected, while teams sponsored by any other company than Adidas, Puma or Nike perform worse. The average advantage per knockout match for the Adidas teams is to raise the probability to win by 10 percent point.
\end{abstract}

Keywords: Jersey sponsorship, advertising, Elo-rating, soccer, football

JEL classification: C53, D72, D73, L82, L83, M30 


\section{Introduction}

The three main sources of finance in association football or soccer are the revenues from the sales of TV rights, gate revenues and revenues from sponsoring and merchandize. In a competition of clubs, such as the English Premier League, there is a two-way causality: more successful clubs receive higher shares of TV revenues, attract more visitors, sell more merchandize and are able to negotiate better sponsor contracts, which allows them to attract better players and supporting staff, making them even more successful (e.g. see Dobson and Goddard 1998). In this paper, contrary to club competitions, there is only a one-way causality since stronger national teams might be able to negotiate better sponsorship contracts, but it is unlikely that better sponsorship contracts enhance the quality of the national team and hence its performance.

We analyze the FIFA World Cup of soccer (hereafter abbreviated as WC) from the perspective of commerce, that of jersey sponsors. As well as a tournament between countries, it can be considered as a tournament between jersey sponsors, which may field one or more teams. The strategies of sponsors can in principle be modeled using the framework of an all-pay auction, where sponsors pay fees and compete for prizes (see e.g. Fang 2002 and Groh et al. 2012). Sponsoring national soccer teams is not a trivial business. The WC is the largest sporting event in the world. The Economist reported in 2010 that the global market for football apparel, shoe and equipment is about $\$ 11$ billion. ${ }^{1}$ Adidas and Nike, the two main rivals in the soccer footwear market, experience a boost in football-related sales just before and during the WC tournaments. Adidas is the Official Licensee and Supplier of the FIFA World Cup and since the 1970 WC it supplies the match balls. The Adidas logo is on the referee uniforms, it has advertisements in the stadiums and its logo on the official FIFA World Cup website. It has also been the sponsor of the German team for nearly five decades since 1954, currently paying \$298 million (\$25.7 million per year ${ }^{2}$ )

\footnotetext{
${ }^{1}$ See http://www.economist.com/node/16486727.

2 “Adidas spends $\$ 125$ million a year on sponsorship deals with the FIFA global league and its six top teams, according to German sports marketing consultant SPORT+MARKT. Nike spends \$75 million per year for the right to sell the game-related gear of five leading teams. The company, sponsor of 10 teams, hopes to expand the Nike brand's $\$ 1.7$ billion soccer business. Adidas had soccer sales of about $\$ 1.8$ billion in 2008” (source:
} 
although Nike placed a rival bid in 2007 of \$648 million for an eight year contract. Nike paid the French football federation, up to 2010 sponsored by Adidas, more than $\$ 500$ million to be its jersey supplier from 2011 up to 2018. ${ }^{3}$ Nike was more active in social media with its campaign "Write the future" in the run-up to the WC2010 and Puma campaigned with the video "Journey of football”. London-based Umbro paid England \$50 million per year in $2010 .^{4}$ No matter how large the sponsor fees may be, due to the one-way relationship running from performance to sponsorship fees but not the other way around as in club competitions where revenues can be used to attract better players, one may expect that the identity of the jersey sponsor does not have any effect on the winning probabilities of national teams.

In this paper two questions are addressed. Firstly, how can the performance of the jersey sponsors on WCs be measured? Obviously, there are many ways in which the performance of sponsors can be measured, e.g. one can count what percentage of goals are scored by Adidas shoes or by teams sponsored by Adidas, but we will focus on the change in ratings of Adidas teams or teams of other sponsors due to their performance on the WC. Secondly, do some sponsors perform significantly better or worse than others? For both questions it is relevant to acknowledge that a sponsor is

http://www.businessweek.com/magazine/content/10_21/b4179022112034.htm)

${ }^{3}$ According to the New York Times (June $6{ }^{\text {th }}$, 2010), the fee to the French soccer federation of Nike’s seven-year deal with France is $€ 40$ million per year against $€ 22$ million for Brazil, while England receives $€ 34$ million for wearing Umbro. Sponsorships fees for the teams in the WC2010 were $€ 85$ million for Adidas, $€ 70$ million for Nike (exclusive of its brand Umbro) and $€ 30$ million for Puma.

(http://www.nytimes.com/2010/06/07/business/global/07ihtsponsor07.html?_r=1\&pagewanted=print)

${ }^{4}$ Puma paid an annual sponsorship fee of $€ 17$ million to Italy, whereas Honduras, sponsored by Spanish Joma, only received $\$ 2$ million. The large difference in sponsorship value is due to differences in performance, economic potential of the country, the importance of football in countries and many other factors (see http://www.wharton.universia.net/index.cfm?fa=viewArticle\&id=1899\&language=english). A major source of revenue for the sponsors are the sale of replica shirts which people wear on the street and replica match balls. Adidas sold more than 6 million shirts in the first quarter of 2010 (see http://internationalsoccer.suite101.com/article.cfm/global-brands-hoping-for-worldcup-glory). 
expected to do better at a tournament the stronger the teams that wear its shirt. Since Brazil and the Netherlands usually perform better than Switzerland and Ghana, it might be expected that a sponsor of Brazil and the Netherlands will do better than a sponsor of Switzerland and Ghana. Therefore, what we want to know is not so much whether some jersey sponsors perform better or worse than other sponsors, but whether some jerseys perform better or worse than expected, that is, controlling for the quality of the teams they sponsor. Thus in a contest between two teams with different sponsors, the difference in team quality must be taken into account. After correcting for the differences in team quality, the default is then that the jersey sponsor is irrelevant for the performance, comparable to any other arbitrary factor, say whether a country name has an even or odd number of letters or syllables. Analogously, in chess it would be strange if the color or brand of the T-shirts or trousers that chess players wear at tournaments turn out to have a significant effect on their performance, on top of the effect of players' strength as measured by their Elo ratings. Instead, one would expect that overall the winning performance of chess players are in line with their Elo-ratings and that really arbitrary characteristics are not significant in explaining the results. In the empirical section, we show that jersey sponsorship has a significant impact on the chances to win, whereas the variables representing arbitrary characteristics such as the odd or even number of letters indeed are highly insignificant.

This research has analogies to studies of discrimination in sports, where the null hypothesis is that race, taking into account talent quality, is not a significant factor of player's salaries (see Berri 1999; Berri and Simmons 2009; Mitchell et al 2011, Pedace 2008). The two performance measures used are the difference in Elorating at the beginning and at the end of the tournament, and the difference between average winning percentage based on actual outcomes versus expected outcomes, both specified by sponsor. The structure of this paper is as follows. In section 2 the Elo-rating methodology is explained. Section 3 describes the data used and the empirical results are presented in section 4. Section 5 summarizes and concludes.

\section{The Elo-rating methodology}


The Elo-rating is used to measure the strength of national teams (see www.eloratings.net). ${ }^{5}$ The alternative for the Elo-rating is the official FIFA-ranking, but not all the specifics to calculate the rankings are revealed by FIFA, although some information of weights used and changes in the ranking method in 1993, 1999 and 2006 has become available (see McHale and Davies 2007). Torgler (2004: 292-3) finds that a higher FIFA-ranking leads to a higher probability to win on the WC 2002 and “... having a referee from the same region has a positive impact on the probability of winning a game. The marginal effects are quite high, but the statistical significance is not robust regarding the estimation method.” Paul and Mitra (2008) also find that higher ranked FIFA teams have higher probabilities to win, using data of the WC 1994-2006. Binder and Findlay (2012, especially Table 1) show that the Elo-ratings outperform the FIFA rankings in forecasting the results of all matches not ending in a draw in the four WC during the period 1994-2006 (see also Lasek et al. (2013) for a similar result). ${ }^{6}$ The Elo-rating however does not outperform predictions based on bookmakers odds, which might be compatible with this study if bookmaker experts consciously or unconsciously include jersey information in their assessment of odds. ${ }^{7}$

In soccer, as well as in almost any other sport, there is considerable uncertainty of outcome. Occasionally, one team might beat a much better team, that is, win a match against a team ranked considerably higher. ${ }^{8}$ To deal with the variability and uncertainty in outcomes, sports statisticians use ratings to assess the strength of teams or players over time. The most famous one is the chess Elo-rating system invented by Arpad Elo in 1959. It assumes that there is a distribution function for each chess player with respect to performance, where the rating $R$ of a player is

\footnotetext{
${ }^{5}$ For more information about the Elo methodology, the relative weight of friendly matches versus matches on the $\mathrm{WC}$ and the equation used to revise the ratings, see www.eloratings.net (sponsored by Advanced Satellite Consulting), especially the section 'About ratings'.

${ }^{6}$ For a systematic approach to evaluate the accuracy of alternative forecasting methods, see Fenton and Constantinou (2010; 2011).

${ }^{7}$ Hvattum and Arntzen (2010) compare ELO-based predictions to six benchmark prediction methods, among which two based on bookmaker odds, and found that ELO outperforms the four benchmarks, but not the bookmakers predictions. Stefani and Pollard (2007) also compare the FIFA- and Elo- rating systems.

${ }^{8}$ For instance, Paul and Mitra (2002) note that none of the top five FIFA-ranked teams reached the semifinals at the WC 2006.
} 
given by the mean of the distribution. Elo originally assumed a normal distribution, but for chess and soccer a logistic distribution is now used, given by the following cumulative distribution function $F$ :

(1) $F(x, R, s)=\frac{1}{e^{-(x-R) / s}+1}$

where $s$ is the standard deviation in performance. This cumulative distribution function gives the probability that the player, given its rating $R$ and the variability $s$ in its performance, performs worse or equal than performance level $x$. The Elo-rating formula used to assess the strengths of national teams and the formula for the expected probability of a win are quite similar, except that the base is changed from $e$ to 10 and $s$ is normalized to 400 . For a match between national teams $\mathrm{A}$ and $\mathrm{B}$, the probability of a win for A is: ${ }^{9}$

(2) $p_{A}^{A B}=\frac{1}{10^{-\left(E L O_{A}-E L O_{B}\right) / 400}+1}$

If the difference in rating is 400 points in A's favor, the probability to win for A is exactly ten times as large as for B (i.e. $1 / 1.1$ or $90.9 \%$ vs. $1 / 11$ or $9.1 \%),{ }^{10}$ where a draw is considered as half a win. A difference of 100 points translates into a $64.0 \%$ chance of victory and 200 points into $76.0 \%$. Figure 1 gives the Elo winning probability curve as a function of the difference in rating. Note that the winning probabilities are determined by the difference in rating and not by the absolute rating levels.

\footnotetext{
${ }^{9}$ With $p$ the probability to win and determined by the difference in Elo-ratings $D$, the log of the so called odds ratio is $\log (p /(1-p))=f(D)$ and solving for $p$ gives $p=\frac{1}{10^{-f(D)}+1}$. The Elo-rating formula is thus a special case of the logistic distribution where $f(D)=D / 400$ and the base $e$ (which would result if instead of Log the Ln function was applied to the odds ratio) is replaced by 10 .

${ }^{10}$ From Eq. (2) it can be derived that the ratio of the probability to win of A and the probability to win of $\mathrm{B}$ is equal to $10^{\left(E L O_{A}-E L O_{B}\right) / 400}$, so a difference in Elo-rating of 400 points translates into a probability to win of A ten times that of B.
} 
Figure 1 about here

After each match, the Elo system calculates the new ratings as follows:

(3) $E L O_{A}^{1}=E L O_{A}^{0}+k\left(w-p_{A}^{A B}\right) \quad w=1($ win $) ; 0.5$ (tie); 0(loss)

with the weighting parameter $k$ dependent on the relative importance of the match (ranging from 20 for friendly matches to 60 for the WC final). To take into account that teams perform better at home, the host team factor is set at +100 Elo-points. Sometimes teams from the same continent are given +50 points (see e.g. Deutsch 2011: 18, Leitner et al. 2010: 474). In this paper, these special adjustments are not made, so it is assumed that all countries in a WC play on neutral ground. No matter what the actual outcome of a match, the zero sum characteristic makes it that the Elopoints won by one team is lost by the other team. A match between A and B results in a change in points for team $\mathrm{A}$ of $k\left(w-p_{A}^{A B}\right)$, whereas for team $\mathrm{B}$ it is $k\left((1-w)-\left(1-p_{A}^{A B}\right)\right)$, so the sum is by definition zero. We can make use of the zero sum nature of the calculation of Elo-ratings after each match, and therefore also before and after the tournament, to measure the performance of different jersey sponsors vis à vis each other to see which jerseys over- and underperform. Therefore, a straightforward way to measure the performance of a jersey sponsor is the change in Elo-rating of its teams before and after the tournament, with an expected change of zero. So if jerseys are an arbitrary factor, then we expect that sponsorship is not systematically related to Elo-points won or lost during the tournament.

The official Elo-ratings just before the start of a WC tournament are used as the indicator of national team quality and hence determine the chances of victory against other teams, using formula (2) above. In principle, since Elo-ratings are updated after every single match, the updated ratings could have been used, but there is a good reason to use the ratings at the start. Suppose that Adidas teams will perform better than expected based on their Elo-ratings at the start of the tournament. Then the overall over-performance of the Adidas teams will gradually be reflected in higher, updated, Elo-ratings. However, by taking the ratings right after the last preparatory friendly match but just before the WC tournament starts, we have an indicator of team 
qualities which is not yet influenced by over- or underperformance at the WC tournament itself.

Given that most teams will have a long term relationship with their jersey sponsor and some jersey sponsors may have a preference for stronger or weaker teams to sponsor, there is not much the sponsor can do to suddenly improve the performance of the team that is not yet included in the Elo rating of the team at the start of the tournament. However, it might be that some sponsors are better able than others to select what may be called 'tournament teams', that is, teams performing significantly better at WC tournaments compared to their performance in between these tournaments. This would imply that for some sponsors and their teams the Elo-ratings at the start are an underestimation of their true strength. Alternatively, because Adidas is the official WC sponsor with advertisements in the stadiums, Adidas teams might feel 'at home' and enjoy a kind of home advantage, so performing better than expected based on their Elo-ratings at the start. Another explanation might be that because the referees are sponsored by Adidas, they might be inclined to give occasionally the benefit of doubt to Adidas teams, e.g. the famous disallowed goal of Lampard in the match between England (sponsored by Umbro) and Germany (Adidas) in 2010. However, to provide a list of plausible and testable explanations or even causal mechanisms how sheer jersey sponsorship exerts a significant impact on team performance is beyond the scope of this paper. The purpose of this paper is more modest, namely to measure the performance of sponsors at WC tournaments and to find out whether or not jersey sponsorship is linked to systematic over- or underperformance of teams.

\section{Data and tournament description}

In the WC 2010 in South Africa, twelve teams were sponsored by Adidas, nine by Nike, seven by Puma and four brands sponsored just one team (England by Umbro, Chile by the US company of running shoes Brooks, Honduras by Joma and North Korea by Legea, an Italian manufacturer of sports equipment). Although Umbro was taken over by Nike in 2007, it is maintained as a separate jersey sponsor in this analysis. Reebok was acquired by Adidas in 2006 and is also maintained as separate. In the group stages, each sponsor hopes that as many of its teams, especially its strongest teams, proceed to the elimination rounds and eventually make it to the final 
and win the WC. The WC tournament starts with a round robin in eight groups, each with four teams. The two top teams in each group advance to the knockout stage, which starts with the round of 16 (also $8^{\text {th }}$ finals, hereafter abbreviated as $8 \mathrm{~F}$ ). The winners in the knockout stages advance to the quarter finals (QF), semi-final (SF) and final respectively.

Table 1 lists the number of teams by jersey sponsor at the last four WCs. Measured by the number of teams, Adidas, besides being official sponsor of the FIFA WC and the official ball sponsor since 1970, is the biggest jersey sponsor overall, followed by Nike and Puma. Aggregated over all four tournaments, we can say there are four sponsors with market shares (in terms of the number of teams sponsored) of around 25\%, where the category 'Other' contains all other sponsors.

Table 1 about here

Since teams differ in Elo-rating, it does matter for which teams the kits are supplied. For the national teams which qualified for the WC, we used the Elo-ratings just before the WC started. For instance, the Elo-rating of France at the WC 2006 is not the updated rating after each match in the WC tournament, nor the rating (2039) it reached after losing the final against Italy, but the rating (1944) right at the start of the tournament (after the last friendly match against China on June $7^{\text {th }}$ 2006).

Table 2 specifies the number of teams together with the average Elo-ratings by sponsor in each stage of the four tournaments, with a panel for each tournament and the fifth panel aggregating all four tournaments. In 2010, the average Elo-rating of the 32 participating teams in the group stage (denoted by G) was 1775 . The nine Nike teams had the highest rating and the seven teams of Puma the lowest rating on average. Out of the twelve Adidas teams the seven that proceeded to the $8 \mathrm{~F}$ had an average Elo-rating of 1836.

Table 2 about here

Only Adidas managed to have a team in the final in all four WCs. Nike managed to do it three times, Puma just once (after it had lost nine of its twelve teams in the group stages of the WC 2006 tournament) and the category of Other sponsors only managed to get a team into the semifinals once. 


\section{Results}

Table 3 lists the countries and sponsors which won the WC or were the losing finalists. The first two columns show that there has not been a final between two teams with the same sponsor. Adidas-Nike occurred thrice and in 2006 it was PumaAdidas, which coincided with Puma being the sponsor with the most teams in the group stage. Adidas managed to have a team in the final on all four occasions. As the last row of Table 1 shows, although most teams are sponsored by companies other than Adidas, Nike or Puma, none of these other sponsors (among them Umbro, Brooks, Lotto, Hummel, Kappa and Reebok) reached the final. Only Lotto (Croatia) reached the semifinals in 1998. Summarizing, the last four WC finals were between teams with different sponsors, the world champion of a tournament is usually sponsored by the biggest sponsor, Adidas made it to the final all four times ${ }^{11}$ and none of the 36 teams sponsored by companies other than Adidas, Nike and Puma reached the final once.

Table 3 about here

To assess how (un)likely this overall picture is, suppose that at random half of the 32 teams are sponsored by company A and the other half by $\mathrm{N}^{12}$ Then there are four possibilities for the final, with chances in between brackets: A-A $((16 / 32) *(15 / 31)=$ $24 \%)$, A-N $((16 / 32) *(16 / 31)=26 \%), N-A(26 \%)$ and N-N (24\%). So with only two equally represented sponsors, one would expect about half of the time a final between the two sponsors and in the other half a final between teams with the same sponsor. It is more in line with the overall picture in Table 1 to assume four equally represented sponsors labeled A, N, P and O. ${ }^{13}$ If teams are only identified by jersey sponsor, for sponsor A we have the four possibilities of a final AA, AN, AP and AO, where the

\footnotetext{
${ }^{11}$ Although the UEFA European championship is not analyzed in this paper, in the last five tournaments Adidas was not only always present in the final, but also delivered the champion each time.

${ }^{12}$ A comparable analysis was done by Wallace and Haigh (2013) to assess the likelihood that the UEFA Champions League draw was exactly the same as at the rehearsal.

${ }^{13}$ As Table 1 shows, there are four sponsors with approximately equal market shares.
} 
chance of AA is slightly below the chance of a final between a team of A and N, because for any A team there are only seven other A teams compared with eight other teams with sponsor N. ${ }^{14}$ The chance of a final AA is $(8 / 32) *(7 / 31)$ or $5.6 \%$, so the chance of a final between two teams with the same sponsor (AA, NN, PP or OO) is then 22.6\%: Given that the chance of a sponsor reaching the final with at least one team is $220 / 496$ or $44 \%$, the chance of a particular sponsor reaching the final four times in a row is $0.44^{4}=3.9 \%$. With four sponsors the chance of having one sponsor present in each of the four finals is $4^{*}(0.44)^{4}=15.5 \%$.

A slightly more precise estimate can be obtained by using the actual number of teams sponsored at each WC, as listed in Table 1. For instance, at the WC2010, the probability of Adidas reaching the final is the sum of the probabilities of the finals AA, A-N, A-P, A-O, N-A, P-A and O-A. The probability of the final A-A, given that Adidas sponsored 12 of the WC2010 teams, is equal to (12/32)*(11/31). The probability of the final A-N is $(12 / 32) *(9 / 31)$, and so on, which gives a total probability of $62 \%$ of an Adidas team in the WC2010 final. Applying the same method for the previous three tournaments gives 35\%, $49 \%$ and $35 \%$, so the chance of Adidas being present in all four finals is $0.62 * 0.35 * 0.49 * 0.35=0.036$ or $3.6 \%$. The chances of being present in all four finals for Nike, Puma and Others is 3,3\%, 1.5\% and 3.4\% respectively, so merely based on the number of teams sponsored, the chance of the same sponsor being present four times in a row is $12 \%$. Maybe at the next WC in Brazil 2014, for the fifth time in a row an Adidas team will reach the final, whereas ex ante the chance of this to happen is very small.

So far, team qualities are not yet taken into account. Figure 2 (upper panel) shows the average Elo-ratings of the teams in the various stages of the tournament. Except for the WC 2002, we see that the average Elo-rating steadily goes up in each successive stage in the tournament. The almost straight line for the WC 2010 implies that the average Elo-rating of the teams increased by almost 70 points per round, due to the successive elimination of the weaker teams in each stage. For WC 2002, see the

\footnotetext{
${ }^{14}$ Alternatively, if each sponsor fields eight teams at random there are 124 possible finals (any team of the first sponsor may encounter one of the 31 other teams, among which 7 with the same sponsor) and the chance of a final between two teams with the same sponsor is $4 * 7 / 124$ or $22.6 \%$. Therefore the probability to have four finals in a row with in each final two different sponsors is $(1-0.226)^{4}=0.359$.
} 
line with diamond-shaped symbols in the upper panel of Figure 2, the deviant decline in the average Elo-rating from the 8F to the quarterfinals and semifinals can entirely be attributed to the exceptional performance of hosting country South Korea (Elorating 1713), eliminating Italy (1978) in the 8F and Spain (1927) in the QF. Using Eq. (2) and the Elo-ratings for Italy and Spain, the expected probability for South Korea to win was only 18\% and 23\%, respectively. Moreover Turkey (Elo-rating 1721), another below average team, ${ }^{15}$ reached the semifinals and ended the tournament playing against South Korea for the third place.

The lower panel of Figure 2 shows the development of average Elo-ratings specified by sponsor, where the data are aggregated over all four WC. For instance, the 33 teams sponsored by Adidas had an average Elo-rating of 1834, against an average Elo-rating for all 128 teams of 1787. The teams sponsored by Puma had on average an Elo-rating of more than 100 points less than the teams sponsored by either Adidas or Nike. As can be seen in Table 2, there were only two teams sponsored by Puma that reached the semifinal and only one (Italy in 2006 with Elo-rating 1912) played in the final.

Figure 2 about here

It is difficult to tell from Figure 2 whether some sponsors do better or worse than expected. Although at each stage the average Elo-ratings of the teams sponsored by Adidas and Nike are above the line for all teams, they also start the tournament with higher than average Elo-ratings, and vice versa for Puma and the other small sponsors. The only conclusion that can be drawn from these figures is that there is a strong tendency that the higher rated teams emerge in the successive stages of the tournament (if the lower rated teams were more successful, the lines would go down). Over-performance is compatible with a flat line, e.g. a sponsor with just two teams which both make it to the final, and even with a declining line, e.g. where the lower rated teams of a sponsor perform better than other teams with the same sponsor.

\footnotetext{
${ }^{15}$ As can be seen in Table 2, the average Elo-rating of all 32 teams of the WC 2002 was 1796. The almost flat profile in Elo-ratings over the various stages of the 2002 tournament is due to the fact that among the four highest rated teams, all with ratings above 1900 (France 2066, Italy 1978, Portugal 1945 and Spain 1927), only Spain reached the quarter finals.
} 
In the introductory section it was argued that if jerseys are arbitrary, combined with the zero sum game of the Elo-ratings, a straightforward way to measure the performance of a jersey sponsor is the change in Elo-rating of its teams before and after the tournament, with an expected change of zero. It turns out that all Adidas teams together have 280 Elo-points more after the four WC tournaments than at the start. The category of Other sponsors teams end up with 286 points less, while the Nike teams gain 39 points in total and the Puma teams lose 33 points. It is however difficult to test whether the observed changes, with an expectation of zero, are statistically significant.

To evaluate whether and to what extent a jersey brand performs better than expected in a statistical sense, we need to define to what extent the actual outcomes can be explained by the qualities of the teams, irrespective of the jersey in which they play. For each match in the knockout phase, we do know the actual outcome (win or loss, denoted by $w$ ) and the expected probability of a win or loss based on the Eloratings (denoted by $p$ and 1-p). Note that if some sponsors or teams have to play a harder schedule, this is taken into account, because if they happen to be pitched against strong opponents, then their probability to win will go down (see Eq. 2, in which the chance to win depends on the difference in rating between the teams). The difference between the actual outcome and the expected probability of that outcome can be considered as over- or underperformance (denoted by $e$ ). So for every match in which a team of sponsor $s$ enters the pitch, we can specify:

(4) $w_{s}=p_{s}+e_{s}$

where $e$ measures the extent to which the actual outcome $w$ cannot be explained by the Elo-difference in strength, which determines $p$. Consider the quarter final match Netherlands (Nike) against Argentina (Adidas) in 1998, which the Netherlands won 21. The Elo-rating for the Netherlands was 1926 and for Argentina was 1873, so based on these ratings the probability of a win for Nike was:

(5) $p_{N}=\frac{1}{10^{-\left(E L O_{N}-E L O_{A}\right) / 400}+1}=\frac{1}{10^{-(1926-1873) / 400}+1}=0.58$ 
and the probability for Adidas to win is $p_{A}=1-p_{N}=0.42$. The actual outcome was a win for Nike, so $w_{N}=1$ and $w_{A}=1-w_{N}=0$, and using Eq. (4) $e_{N}=0.42$ and $e_{A}=-0.42$. Note that each match with two different sponsors generates two observations for the regression analysis ${ }^{16}$ (see below) of Eq. (4), here (w; p; e) equal to $(1 ; 0.58 ; 0.42)$ and $(0 ; 0.42 ;-0.42)$ respectively.

Table 4 gives an overview of the results for the WC 1998; the last three columns specify the over- and underperformance by sponsor. There was only one knockout match, Brazil vs. Netherlands, involving the same sponsor. As can be seen from the bottom row, on average Adidas, Nike and the category of other sponsors score as expected, with only a small advantage for Adidas and the category of other sponsors and a small disadvantage for Nike.

Table 4 about here

Table 5 summarizes the results for all four tournaments, based on similar calculations as spelled out in Table 4. The neutrality of sponsors in 1998 does not hold for the last three WCs. Adidas performs much better than expected in 2006 and 2010 (an extra 0.20 and 0.28 of a win per match on average), while the category of other sponsors performs much worse than expected in 2002, 2006 and 2010. For Puma and Nike the results are mixed, sometimes performing better or worse than expected. For instance, Nike performed worse than expected in the last two tournaments, but it did much better (an extra 0.25 win per match) in the 2002 tournament, in which it also delivered the champion (Brazil).

Table 5 about here

The 2010 tournament shows the largest difference between actual and expected outcomes, differentiated by sponsor. In 2010, seven out of twelve Adidas-teams survived the group stages (see Table 2). Adidas was represented in 10 of the 16 knockout matches, but in five of them the match was between two Adidas teams (see Table 6, which gives an overview of all knockout matches in 2010). In the other five

\footnotetext{
${ }^{16}$ In the analysis of Torgler (2004), the 63 matches of the WC2002 also generate double the amount of observations.
} 
matches where Adidas met teams with rival sponsors, they won four times, which gives an average winning percentage of 0.80 (see the column $w$ for Adidas in Table 5). The expected winning percentage for these five matches was only 0.52 , so the Adidas teams performed much better, on average 0.28 of a win per match, than expected based on their and the opponents Elo-ratings.

Table 6 about here

The over-performance of Adidas in terms of a higher than expected winning percentage can be translated into equivalent Elo-ratings. Using the information in Table 6, it can be calculated that the average Elo-rating of the Adidas teams in the five matches against rival teams (Germany vs. England, Netherlands vs. Slovakia, Spain vs. Portugal, Germany vs. Uruguay and Spain vs. Netherlands) happens to be equal to the average rating of their opponents (1934). Using the Elo win probability formula of equation (2), to raise the expected winning percentage from 0.52 to 0.80 when playing a 1934-rated team ${ }^{17}$ requires an Elo-rating of 2174. The 240 Elo-points extra is more than two times higher than the standard 100 Elo-points extra to account for home advantage. Admittedly, the over-performance of the Adidas teams in the WC 2010 knockout phase was exceptional. However, as can be seen in the last two rows of Table 5, Adidas teams performed better than expected by 0.10 of a win per match on average, calculated over all 32 matches in the four tournaments where it met a rival team in the knockout phase. Also the Puma teams enjoyed an advantage of 0.10 win per match over the 11 knockout matches against rival sponsors. Instead of an expected win probability of 0.42 over 23 matches, the actual winning percentage for the category of other sponsors is only 0.26 .

Using the Elo win probability function, the equivalent number of the extra Elo-points required (denoted by $\Delta$ ) to raise the expected probability of winning by a small margin (denoted by $\delta$ ) can be calculated as follows.

\footnotetext{
${ }^{17}$ If a team with an Elo-rating of 2000 plays three matches against teams with Elo-ratings of 2550, 1800 and 1800, then its expected win percentages are 4\%, 76\% and 76\% respectively. Therefore its average win percentage is $52 \%$, despite the fact that its Elo-rating is below the average Elo-rating of the opponents (2000 vs. 2050). As can be seen from Figure 1, there is a decreasing marginal probability to win built into the Elo-rating methodology.
} 
(6a) $p=\frac{1}{10^{-\left(E L O_{A}-E L O_{R}\right) / 400}+1} \Rightarrow 10^{-\left(E L O_{A}-E L O_{R}\right) / 400}=\frac{1}{p}-1 \Rightarrow E L O_{A}-E L O_{R}=-400 * \log \left(\frac{1}{p}-1\right)$

(6b) $p+\delta=\frac{1}{10^{-\left(E L O_{A}+\Delta-E L O_{R}\right) / 400}+1} \Rightarrow E L O_{A}+\Delta-E L O_{R}=-400 * \log \left(\frac{1}{p+\delta}-1\right)$

Subtracting gives:

(7) $\Delta=400 * \log \left(1+\frac{\delta}{p(1-p-\delta)}\right)$

Using Eq. (7), the extra 0.10 wins per match of Adidas (raising the expected win probability from 0.56 to 0.66 ) is equivalent to an extra Elo-rating of 73 points for every Adidas team entering the pitch in the knockout phase against a team with a rival sponsor. For Puma this figure is 70 points and for the category of other sponsors it is 126 points. Given that the standard adjustment for playing at home is 100 Elo-points, playing in Adidas or Puma shirts may give a similar feeling to that of playing at home. As noted before, there might be a beneficial psychological effect from Adidas being the official WC sponsor and ball supplier on the Adidas teams. Teams with a jersey belonging to the category Other experience on average a disadvantage which is even higher than playing an away match.

To find out whether the effects of jersey sponsor on outcomes are significant, a regression analysis was performed. ${ }^{18}$ In the first, the measure of over- or underperformance (e) that cannot be explained by the Elo-ratings (see Eq. 4 above) has been run on dummies for jersey sponsor and the results are listed in Table 7. In total there are 49 knockout matches with different sponsors, which gives 98 observations. All effects of the dummy variables Adidas $(A)$, Nike $(N)$ or Puma $(P)$ are relative to the category of other sponsors $(O)$. Adidas turns out to have a statistically significant effect, raising the win probability by 0.26 relative to the

\footnotetext{
${ }^{18}$ Hoffman et al (2002) perform a regression analysis in which they try to explain the FIFAranking by variables such as GNP per capita, population size, temperature in the capital and a dummy Latin for a strong cultural predisposition to football.
} 
category of other sponsors. ${ }^{19}$ The coefficients for Nike and Puma are also positive, but not significant.

Tables 7 and 8 about here

Not shown in the tables, but adding dummies for each WC does not deliver any additional significant results. Since for each of the 49 knockout matches between different sponsors we have two observations, we can add a dummy variable for the team which is mentioned first, so playing 'at home', although at the WC tournament there is no real home advantage for the team mentioned first (for an overview of the different causes of home advantage, see Pollard 2008). As expected, this dummy variable turns out to be insignificant. Inclusion of other variables based on arbitrary characteristics - one dummy variable based on the first letter of the country name playing 'at home’ (Oddh) or 'away' (Odda), coded odd when starting with A,C, E, etc. and even when starting with B, D, F, etc., another one based on the number of letters in the country name, which can be odd or even (Oddletters), or based on an even or odd number of vowels in the country name (Oddvowels) - all turned out to be highly insignificant, which strengthens the finding that jersey sponsorship is not an entirely arbitrary factor in knockout matches at the last four WC tournaments.

In the second regression, see Table 8 , the actual outcome $w$, which is either 1 or 0, is regressed on the expected Elo-probability to win $(p)$ and dummies for the jersey sponsor (see Eq. 4). Here again only Adidas is statistically significant. This result does not change if instead of ordinary least squares a probit regression is performed. The coefficient for the Elo-probability to win is highly significant and equal to 0.87 , which is close to the expected value of unity. The lower panel of Table 8 shows the results of a univariate regression of $w$ on $p$ only, which gives as expected a zero value for the intercept and a value close to unity for the variable representing the Elo-probability to win. Both regressions in Table 8 have highly significant F-tests.

\footnotetext{
${ }^{19}$ Note that the advantage of Adidas relative to the category of other sponsors, of 0.26 per match, is consistent with the outcomes in the last row of Table 5. Averaging across all four tournaments, Adidas had an advantage of 0.10 wins per match and the category Other a disadvantage of -0.16 , so the relative advantage of Adidas versus Other is 0.26 .
} 
Thus, the main finding in this paper is that Adidas teams do better than expected based on their ELO ratings in the knockout matches of the last four WCs, but it is yet unclear what the causal mechanisms are of wearing a specific jersey on team success. Firstly, Adidas is the main official sponsor of the FIFA WC, so one mechanism might be that Adidas-sponsored teams experience a 'home advantage' effect, while team wearing jerseys of other sponsors suffer an 'away disadvantage'. A second explanation is that Adidas is simply better at picking successful teams that 'play above their heads' in tournaments (e.g. Germany has a reputation to be a strong tournament team), so the Elo-ratings of Adidas teams at the start of the tournament will be underestimating their real strength. Thirdly, Adidas may provide more and better resources (sports equipment, training facilities) to its teams or pay them higher performance fees. Fourth, since the Adidas-logo is on the referee uniforms, there might also be a referee bias in favour of Adidas-sponsored teams. If referees can build a better career if they gives Adidas teams the benefit of doubt in case of decisions that can go either way (penalty or not, red cards, (dis)allowance of goals), then this referee bias $^{20}$ can be explained in a rational choice framework. ${ }^{21}$ Fifth, taking into account that the dummy variable for Adidas is just significant at the 95\%-level, we must leave open the possibility that even if sponsorship has no effect at all on the outcome, the observed result is got by chance. Relevant in this respect is that although the Elorating is a good predictor of the quality of a team over a large number of matches, it can be a poor predictor for a single match. The Adidas effect might also be due to an omitted variable bias, if variables left out correlate in some systematic way with jersey sponsorship.

\footnotetext{
${ }^{20}$ In terms of Walsh and Giulianotti (2001: 66), this would fall into the category of corrosion of the integrity of the game due to the commodification of football.

${ }^{21}$ A rational choice approach to the referee bias, focusing on the career prospects of referees, would run as follows. Referees want to make a career, for instance they strife to be selected for the WC and assigned important matches, such as the WC final. Referees that signal that they understand the game, in this context occasionally giving the benefit of doubt to an Adidas team, will have a higher probability to be selected for the WC. The advantage of this approach is that there is no need for a complot theory, i.e. that a referee receives strict orders to manipulate a match.
} 


\section{Summary and conclusion}

The market for soccer jerseys, shoes and equipment is a multi-billion market, in which the three major companies are Adidas, Nike and Puma. One way to increase sales and protect or raise the market share is to sponsor national teams. Contrary to matches in club competitions, at WC tournaments no other branding than the logos of the jersey sponsor on the uniform is allowed. Instead of a two-way causality as in league competitions, there is only a one-way causality for national teams, where stronger performing national teams receive higher sponsorship fees than others, but neither type of sponsors nor the fee itself should have an impact on the performance, on the same footing that brands of cloths are unlikely to have a significant impact on the performance of chess players.

The main focus in this paper was to investigate whether or not jersey sponsors have an effect on the outcome of WC knockout matches, taking team qualities into account. Team qualities were measured by the Elo-ratings of national soccer teams at the start of the tournament. Some teams may perform better or worse than expected based on their Elo-rating, but due to the zero sum nature of the Elo- rating system all teams together by definition have the same total of Elo-points at the end of the tournament as at the start. In principle, this should also apply for each sponsor separately, provided each sponsor field a sufficiently large number of teams so that the law of large numbers applies. Adidas, Nike and Puma each had around 30 teams at the last four WCs, and the other smaller sponsors where merged into a group containing 36 teams. The Adidas teams together won 280 Elo-points, whereas the teams with a small sponsor lost 286 points. Nike won 39 and Puma lost 33 points. Based on these numbers, it is hard to conclude that Adidas teams significantly overperform on WCs. For this reason, a regression analysis was conducted. In one specification the effect of jersey brands on over-or underperformance was estimated. In the other, almost equivalent, specification the actual outcomes in terms of wins or losses were related to the probability of winning, as determined by the difference in Elo-ratings, and dummies for jersey brands. Both estimations show that Adidas teams perform significantly better than expected based on their and their opponents Eloratings at the start of the tournaments. Expressed in Elo-points, the average advantage for an Adidas team is equivalent to an extra 73 points, amounting to an increase in the probability to win of 0.10 . The disadvantage for the teams merged in the category of Other sponsors is on average 126 Elo-points, lowering the probability to win by 0.16 . 
To put this into perspective, the standard adjustment for home advantage is 100 Elopoints, raising the win probability by 0.14 .

The analysis in this paper was based on comparing actual outcomes and probabilities to win determined by Elo-rating differentials. Further research has to show whether alternative specifications of the ex ante probability to win lead to the same results as those generated by using Elo-ratings. Additionally, more attention to potentially statistical problems such as the omitted variable bias is required to rule out spurious results. If the results do indeed hold, then it becomes interesting to identify which causal factors might drive these results, an effort not pursued in this contribution. Another avenue for further research is to investigate the effect of jersey sponsors at other country tournaments, such as the European, African or South American championships, or in national and international club competitions.

\section{References}

Berri, D.J. (1999), Who is 'most valuable'? Measuring the player's production of wins in the National Basketball Association, Managerial and Decision Economics, Vol 20 (8): 411-27.

Berri, D.J. and R. Simmons (2009), Race and the Evaluation of Signal Callers in the National Football League, Journal of Sports Economics, 10 (1), 23-43.

Binder, J.J. and M. Findlay (2012), The Effects of the Bosman Ruling on National and Club Teams in Europe, Journal of Sports Economics, 13 (2), 107-29.

Constantinou, A. and N.E. Fenton (2010), Evaluating the Predictive Accuracy of Association Football Forecasting Systems, working paper, University of London.

Constantinou, A. and N.E. Fenton (2011), Solving the problem of inadequate scoring rules for assessing probabilistic football forecast models, University of London [Submitted for publication in the Journal of the Royal Statistical Society, Series C: Applied Statistics, August 2011]

Deutsch, C. (2011), Looking Back at South Africa: Analyzing and Reviewing the 2010 FIFA World Cup, Chance 24 (2), 15-23.

Dobson, S.M. and J.A. Goddard (1998), Performance and revenue in professional league football: Evidence from Granger causality tests, Applied Economics, vol. 30(12), 1641-1651. 
EloRatings.net (2012), ‘The World Football Elo Rating System’. http://www.eloratings.net/system.htm.

Fang, H. (2002), Lottery versus all-pay auction models of lobbying, Public Choice 112: 351-71.

Groh, C., B. Moldovanu, A. Sela and U. Sunde (2012), Optimal Seedings in Elimination Tournaments, Economic Theory, vol. 49(1), 59-80.

Hvattum, L.M. and H. Arntzen (2010), Using ELO Ratings for Match Result Prediction in Associations Football, International Journal of Forecasting 26, 460-470.

Hofmann, R., C. Lee and B. Ramasamy (2002), The Socio-economic Determinants of International Soccer Performance, Journal of Applied Economics V (2), 253-72.

Lasek, J., Z. Szlávik and S. Bhulai (2013), The predictive power of ranking systems in association football, International Journal of Applied Pattern Recognition, 1 (1), 27-46.

Leitner, C., A. Zeileis and K. Hornik (2010), Forecasting Sports Tournaments by Ratings of (Prob)abilities: A Comparison for the EURO 2008, International Journal of Forecasting 26, 471-481.

McHale, I. and Davies, S. (2007), Statistical analysis of the effectiveness of the FIFA World Rankings, in J. Albert and R. Koning, eds, 'Statistical Thinking in Sports', Chapman \& Hall/CRC, Boca Raton, Florida, pp. 77-90.

Mitchell, H., C. Stavros and M. Stewart (2011), Does the Australian Football League Draft Undervalue Indigenous Australian Footballers?, Journal of Sports Economics, 12 (1), 36-54.

Paul, S. and R. Mitra (2008), How Predictable are the FIFA Worldcup football outcomes? An Empirical Analysis, Applied Economic Letters, 15 (15), 1171-6.

Pedace, R. (2008), Earnings, Performance, and Nationality Discrimination in a Highly Competitive Labor Market as An Analysis of the English Professional Soccer League, Journal of Sports Economics, 9 (2), 115-140.

Pollard, R. (2008), Home advantage in football: A current review of an unsolved puzzle, The Open Sports Sciences Journal 1(1), 12-14.

Stefani, R. and R. Pollard (2007), Football rating systems for top-level competition: A critical survey, Journal of Quantitative Analysis in Sports 3, Article 3.Torgler, B. (2004), The Economics of the FIFA Football Worldcup, Kyklos 57 (2), 287300. 
Wallace, M. and J. Haigh (2013), Football and Marriage - and the UEFA draw, Significance 10 (2), 47-8.

Walsh, A. and R. Giulianotti (2001), This Sporting Mammon: A Normative Critique of the Commodification of Sport, Journal of the Philosophy of Sport, XXVIII, 53-77.

Table 1. Number of teams sponsored, WC 1998-2010

\begin{tabular}{cccccc}
\hline WC & Adidas & Nike & Puma & Other & Total \\
\hline 2010 & 12 & 9 & 7 & 4 & 32 \\
2006 & 6 & 8 & 12 & 6 & 32 \\
2002 & 9 & 8 & 3 & 12 & 32 \\
1998 & 6 & 6 & 6 & 14 & 32 \\
Total & 33 & 31 & 28 & 36 & 128 \\
\hline
\end{tabular}


Table 2. Number of team per sponsor and average Elo-ratings, by tournament and stage $(\mathrm{G}=$ group stage; $8 \mathrm{~F}=$ eight finals; $\mathrm{QF}=$ quarter finals; $\mathrm{SF}=$ semifinals; $\mathrm{TP}=$ third place; $\mathrm{F}=$ final).

\begin{tabular}{cccccccccccc}
\hline 2010 & Adidas & Nike & Puma & Other & total & 2006 & Adidas & Nike & Puma & Other & total \\
G & 12 & 9 & 7 & 4 & 32 & G & 6 & 8 & 12 & 6 & 32 \\
Elo & 1785 & 1803 & 1730 & 1762 & 1775 & Elo & 1829 & 1849 & 1723 & 1779 & 1785 \\
8F & 7 & 5 & 2 & 2 & 16 & $8 F$ & 4 & 5 & 3 & 4 & 16 \\
Elo & 1836 & 1893 & 1752 & 1914 & 1853 & Elo & 1913 & 1893 & 1763 & 1802 & 1851 \\
QF & 4 & 2 & 2 & & 8 & QF & 3 & 2 & 1 & 2 & 8 \\
Elo & 1915 & 2052 & 1752 & & 1908 & Elo & 1905 & 1949 & 1912 & 1849 & 1903 \\
SF & 2 & 1 & 1 & & 4 & SF & 2 & 1 & 1 & & 4 \\
Elo & 2007 & 2016 & 1819 & & 1962 & Elo & 1908 & 1887 & 1912 & 1904 \\
TP & 1 & & 1 & & 2 & TP & 1 & 1 & & 2 \\
Elo & 1929 & & 1819 & & 1874 & Elo & 1872 & 1887 & & 1880 \\
F & 1 & 1 & & & 2 & F & 1 & & 1 & & 2 \\
Elo & 2085 & 2016 & & & 2051 & Elo & 1944 & & 1912 & & 1928
\end{tabular}

\begin{tabular}{cccccccccccc}
2002 & Adidas & Nike & Puma & Other & total & 1998 & Adidas & Nike & Puma & Other & total \\
G & 9 & 8 & 3 & 12 & 32 & G & 6 & 6 & 6 & 14 & 32 \\
Elo & 1836 & 1806 & 1736 & 1775 & 1796 & Elo & 1936 & 1845 & 1672 & 1760 & 1792 \\
8F & 5 & 4 & 1 & 6 & 16 & $8 \mathrm{~F}$ & 5 & 4 & & 7 & 16 \\
Elo & 1828 & 1785 & 1705 & 1841 & 1814 & Elo & 1923 & 1900 & 1823 & 1873 \\
QF & 3 & 3 & & 2 & 8 & QF & 3 & 3 & 2 & 8 \\
Elo & 1827 & 1778 & & 1797 & 1801 & Elo & 1952 & 1973 & 1828 & 1929 \\
SF & 2 & 2 & & & 4 & SF & 1 & 2 & 1 & 4 \\
Elo & 1777 & 1778 & & & 1777 & Elo & 1963 & 1995 & 1828 & 1945 \\
TP & 1 & 1 & & & 2 & TP & & 1 & 1 & 2 \\
Elo & 1721 & 1713 & & & 1717 & Elo & & 1926 & 1828 & 1877 \\
F & 1 & 1 & & & 2 & F & 1 & 1 & & & 2 \\
Elo & 1833 & 1842 & & & 1838 & Elo & 1963 & 2064 & & 2014 \\
\hline
\end{tabular}

\begin{tabular}{lrrrrr}
\hline All WC & Adidas & Nike & Puma & Other & total \\
G & 33 & 31 & 28 & 36 & 128 \\
Elo & 1834 & 1824 & 1715 & 1768 & 1787 \\
8F & 21 & 18 & 6 & 19 & 64 \\
Elo & 1869 & 1870 & 1750 & 1834 & 1848 \\
QF & 13 & 10 & 3 & 6 & 32 \\
Elo & 1901 & 1925 & 1805 & 1824 & 1885 \\
SF & 7 & 6 & 2 & 1 & 16 \\
Elo & 1907 & 1908 & 1866 & 1828 & 1897 \\
TP & 3 & 3 & 1 & 1 & 8 \\
Elo & 1841 & 1842 & 1819 & 1828 & 1837 \\
F & 4 & 3 & 1 & 0 & 8 \\
Elo & 1956 & 1974 & 1912 & 0 & 1957 \\
\hline & & & & &
\end{tabular}

Table 3. Finalists and main sponsors

\begin{tabular}{lllll}
\hline WC & Champion & Finalist & Biggest sponsor & 2nd sponsor \\
$\mathbf{2 0 1 0}$ & Adidas (Spain) & Nike (Netherlands) & Adidas & Nike \\
$\mathbf{2 0 0 6}$ & Puma (Italy) & Adidas (France) & Puma & Nike \\
$\mathbf{2 0 0 2}$ & Nike (Brazil) & Adidas (Germany) & Adidas & Nike \\
$\mathbf{1 9 9 8}$ & Adidas (France) & Nike (Brazil) & Other & Nike \\
\hline
\end{tabular}


Table 4. Over- and underperformance specified by sponsor, WC 1998

\begin{tabular}{|c|c|c|c|c|c|c|c|c|c|c|}
\hline 1998 & Country & Sponsor & ELO & Country & Sponsor & ELO & Outcome & $e_{A}$ & $e_{N}$ & $e_{O}$ \\
\hline $8 \mathrm{~F}$ & Italy & Nike & 1929 & Norway & Umbro & 1878 & $1-0$ & & 0,43 & $-0,43$ \\
\hline $8 F$ & Brazil & Nike & 2064 & Chile & Reebok & 1757 & $4-1$ & & 0,15 & $-0,15$ \\
\hline $8 F$ & France & Adidas & 1963 & Paraguay & Reebok & 1708 & $1-0$ & 0,19 & & $-0,19$ \\
\hline $8 F$ & Nigeria & Nike & 1680 & Denmark & Hummel & 1827 & $1-4$ & & $-0,30$ & 0,30 \\
\hline $8 F$ & Germany & Adidas & 2021 & Mexico & Aba-Spor & 1825 & $2-1$ & 0,24 & & $-0,24$ \\
\hline $8 F$ & Netherlands & Nike & 1926 & Yugoslavia & Adidas & 1934 & $2-1$ & $-0,51$ & 0,51 & \\
\hline $8 F$ & Romania & Adidas & 1825 & Croatia & Lotto & 1828 & $0-1$ & $-0,50$ & & 0,50 \\
\hline $8 \mathrm{~F}$ & Argentina & Adidas & 1873 & England & Umbro & 1937 & $2-2$ & 0,59 & & $-0,59$ \\
\hline QF & Italy & Nike & 1929 & France & Adidas & 1963 & $0-0$ & 0,45 & $-0,45$ & \\
\hline QF & Brazil & Nike & 2064 & Denmark & Hummel & 1827 & $3-2$ & & 0,20 & $-0,20$ \\
\hline QF & Netherlands & Nike & 1926 & Argentina & Adidas & 1873 & $2-1$ & $-0,42$ & 0,42 & \\
\hline QF & Germany & Adidas & 2021 & Croatia & Lotto & 1828 & $0-3$ & $-0,75$ & & 0,75 \\
\hline SF & Brazil & Nike & 2064 & Netherlands & Nike & 1926 & $1-1$ & & & \\
\hline SF & France & Adidas & 1963 & Croatia & Lotto & 1828 & $2-1$ & 0,31 & & $-0,31$ \\
\hline TP & Netherlands & Nike & 1926 & Croatia & Lotto & 1828 & $1-2$ & & $-0,64$ & 0,64 \\
\hline$F$ & France & Adidas & 1963 & Brazil & Nike & 2064 & $3-0$ & 0,64 & $-0,64$ & \\
\hline Avg & & & & & & & & 0,02 & $-0,04$ & 0,01 \\
\hline
\end{tabular}

Table 5. Summary of average actual and expected winning percentage, and over- and underperformance specified by sponsor and tournament

\begin{tabular}{|c|c|c|c|c|c|c|c|c|c|c|c|c|}
\hline & \multicolumn{3}{|c|}{ Adidas } & \multicolumn{3}{|c|}{ Nike } & \multicolumn{3}{|c|}{ Puma } & \multicolumn{3}{|c|}{ Other } \\
\hline & w & $\mathrm{p}$ & e & w & $\mathrm{p}$ & e & w & $\mathrm{p}$ & e & w & $\mathrm{p}$ & e \\
\hline 2010 & 0,80 & 0,52 & 0,28 & 0,43 & 0,58 & $-0,15$ & 0,50 & 0,39 & 0,11 & 0,00 & 0,39 & $-0,39$ \\
\hline 2006 & 0,71 & 0,52 & 0,20 & 0,29 & 0,51 & $-0,23$ & 0,67 & 0,50 & 0,17 & 0,25 & 0,45 & $-0,20$ \\
\hline 2002 & 0,60 & 0,59 & 0,01 & 0,67 & 0,41 & 0,25 & 0,00 & 0,32 & $-0,32$ & 0,17 & 0,52 & $-0,35$ \\
\hline 1998 & 0,60 & 0,58 & 0,02 & 0,56 & 0,59 & $-0,04$ & & & & 0,36 & 0,36 & 0,01 \\
\hline All & 0,66 & 0,56 & 0,10 & 0,50 & 0,52 & $-0,02$ & 0,55 & 0,44 & 0,10 & 0,26 & 0,42 & $-0,16$ \\
\hline \multicolumn{2}{|c|}{$\mathrm{N}$ matches } & \multicolumn{2}{|c|}{32} & & \multicolumn{2}{|l|}{32} & \multicolumn{3}{|c|}{11} & \multicolumn{3}{|c|}{23} \\
\hline
\end{tabular}


Table 6. Knockout phase WC 2010, countries, sponsors, Elo-ratings and outcomes.

\begin{tabular}{clllllll} 
2010 & Country & Sponsor & Elo & Country & Sponsor & Elo & Outcome \\
\hline 8F & Uruguay & Puma & 1819 & Korea & Nike & 1748 & $2-1$ \\
8F & USA & Nike & 1765 & Ghana & Puma & 1685 & $1-2$ \\
8F & Germany & Adidas & 1929 & England & Umbro & 1974 & $4-1$ \\
8F & Argentina & Adidas & 1899 & Mexico & Adidas & 1883 & $3-1$ \\
8F & Netherlands & Nike & 2016 & Slovakia & Adidas & 1643 & $2-1$ \\
8F & Brazil & Nike & 2088 & Chile & Brooks & 1864 & $3-0$ \\
8F & Paraguay & Adidas & 1745 & Japan & Adidas & 1667 & $0-0$ \\
8F & Spain & Adidas & 2085 & Portugal & Nike & 1846 & $1-0$ \\
QF & Brazil & Nike & 2088 & Netherlands & Nike & 2016 & $1-2$ \\
QF & Uruguay & Puma & 1819 & Ghana & Puma & 1685 & $1-1$ \\
QF & Argentina & Adidas & 1899 & Germany & Adidas & 1929 & $0-4$ \\
QF & Paraguay & Adidas & 1745 & Spain & Adidas & 2085 & $0-1$ \\
SF & Netherlands & Nike & 2016 & Uruguay & Puma & 1819 & $3-2$ \\
SF & Germany & Adidas & 1929 & Spain & Adidas & 2085 & $0-1$ \\
TP & Germany & Adidas & 1929 & Uruguay & Puma & 1819 & $3-2$ \\
F & Spain & Adidas & 2085 & Netherlands & Nike & 2016 & $1-0$
\end{tabular}

Table 7. Regression of over- or underperformance on jersey sponsors, $e$ dependent variable.

\begin{tabular}{|c|c|c|c|c|c|c|}
\hline$e$ & Coeff. & Std.Err. & t Stat & $P$-value & \multicolumn{2}{|c|}{ 95\% Conf. Interval } \\
\hline Intercept & $-0,16$ & 0,10 & $-1,62$ & 0,11 & $-0,35$ & 0,03 \\
\hline A & 0,26 & 0,13 & 2,02 & 0,05 & 0,004 & 0,51 \\
\hline $\mathrm{N}$ & 0,14 & 0,13 & 1,07 & 0,29 & $-0,12$ & 0,39 \\
\hline $\mathrm{P}$ & 0,26 & 0,17 & 1,52 & 0,13 & $-0,08$ & 0,59 \\
\hline Oddh & 0,07 & 0,11 & 0,69 & 0,49 & $-0,14$ & 0,29 \\
\hline Odda & $-0,06$ & 0,10 & $-0,56$ & 0,58 & $-0,25$ & 0,14 \\
\hline Oddvowels & $-0,04$ & 0,10 & $-0,45$ & 0,66 & $-0,24$ & 0,15 \\
\hline Oddletters & 0,06 & 0,10 & 0,58 & 0,56 & $-0,14$ & 0,26 \\
\hline
\end{tabular}

Table 8. Regression of outcomes on probability to win and jersey sponsors, $w$ dependent variable.

\begin{tabular}{|c|c|c|c|c|c|c|}
\hline w & Coeff. & Std. Err. & t Stat & $P$-value & \multicolumn{2}{|c|}{ 95\% Conf. Interval } \\
\hline Intercept & $-0,10$ & 0,15 & $-0,69$ & 0,49 & $-0,40$ & 0,19 \\
\hline $\mathrm{pr}$ & 0,87 & 0,27 & 3,23 & 0,00 & 0,34 & 1,41 \\
\hline A & 0,27 & 0,13 & 2,06 & 0,04 & 0,01 & 0,54 \\
\hline $\mathrm{N}$ & 0,15 & 0,13 & 1,15 & 0,25 & $-0,11$ & 0,41 \\
\hline$P$ & 0,26 & 0,17 & 1,53 & 0,13 & $-0,08$ & 0,60 \\
\hline
\end{tabular}

\begin{tabular}{lrrrrrr}
\hline \multicolumn{1}{c}{$w$} & Coeff. & Std.Err. & \multicolumn{1}{c}{$t$ Stat } & $P$-value & & \multicolumn{2}{c}{$95 \%$ Conf. Interval } \\
\cline { 1 - 5 } Intercept & 0,00 & 0,14 & $-0,02$ & 0,98 & $-0,28$ & 0,27 \\
$\mathrm{pr}$ & 1,01 & 0,26 & 3,88 & 0,00 & 0,49 & 1,52 \\
\hline
\end{tabular}


Figure 1. The Elo winning probability curve as a function of the difference in rating

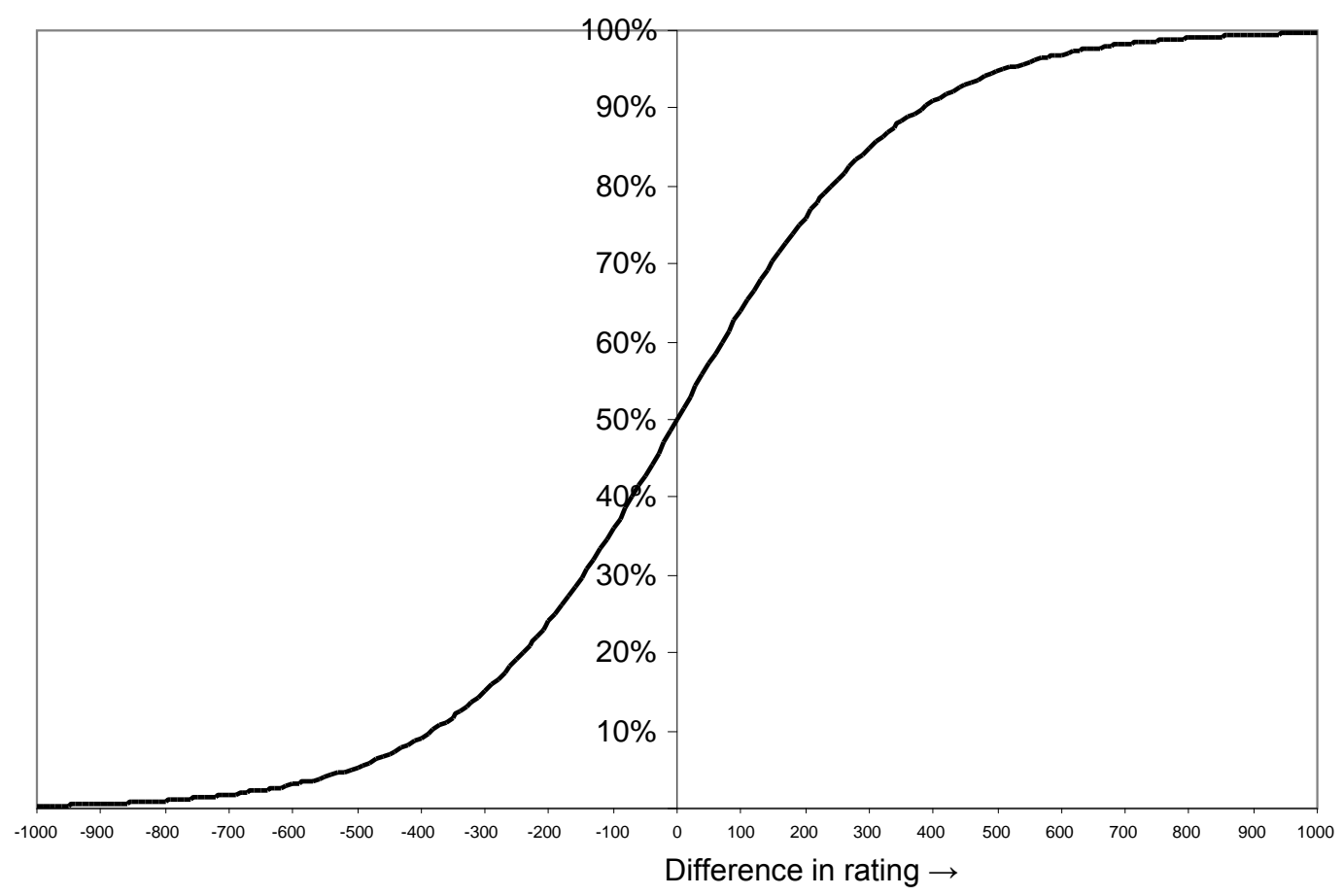



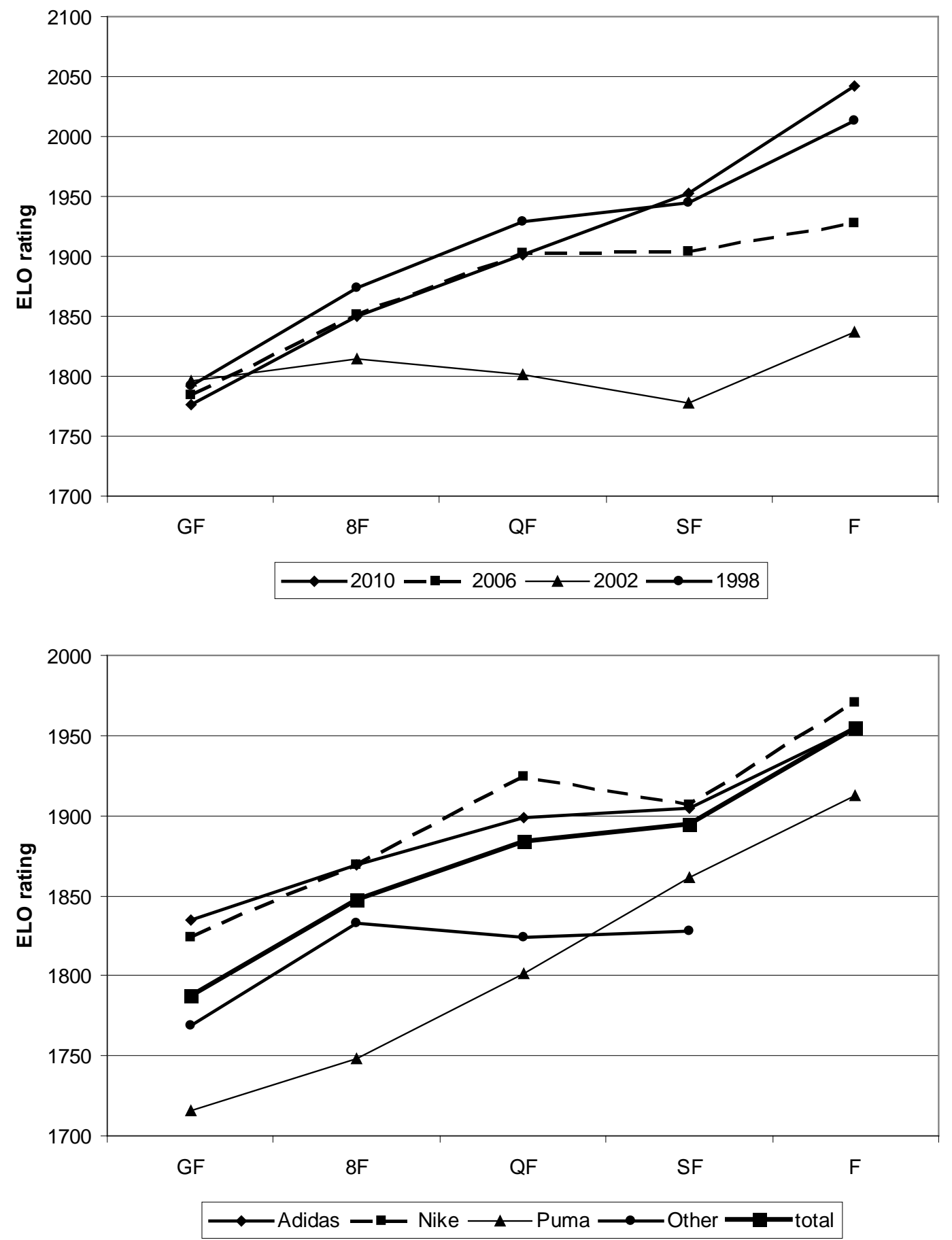

Figure 2. Average Elo-ratings by stage, specified by tournament (upper panel) and by sponsor (lower panel). 